\title{
Determinación de la demanda insatisfecha de miel de abeja en el cantón Morona, provincia de Morona Santiago.
}

\begin{abstract}
Determination of the unsatisfied demand for honey in the Morona canton, Morona Santiago province.
\end{abstract}

Edison Ruperto Carrillo Parra. ${ }^{1}$, Luis Abdón Rojas Oviedo. ${ }^{2}$ \& Tamia Elizabeth Noboa Abdo. ${ }^{3}$

\begin{abstract}
.
Introduction. Bee honey is an ancient product that has been part of human nutrition and other therapeutic uses, it is made up of sugars (fructose, glucose, sucrose), amino acids and minerals, which has made it a palatable food for man and some wild animals; Throughout human evolution, different ways have been sought to efficiently take advantage of this resource, which today has become a real business opportunity, especially in the rural sector, a space that provides ecological facilities and where sources of work and subsistence every day they are more scarce. Objective. In this context, the objective of this study was to determine the unsatisfied demand for honey in the Morona canton, Morona Santiago province, the document consists of, this objective tries to

1 Escuela Superior Politécnica de Chimborazo, Sede Morona Santiago, Macas, Ecuador, edison.carrillop@espoch.edu.ec https://orcid.org/0000-0002-8860-1278

2 Escuela Superior Politécnica de Chimborazo, Sede Morona Santiago Macas, Ecuador. luis.rojaso@espoch.edu.ec, httpss://orcid.org/0000-0002-6424-1642

3 Escuela Superior Politécnica de Chimborazo, Sede Morona Santiago Macas, Ecuador, tnoboa@espoch.edu.ec https://orcid.org/0000-0001-9815-0477
\end{abstract}


respond to the problem statement. Methodology. Part of a qualitative and quantitative approach, based on the inductive and deductive method, the population sample consisted of 372 households in the Morona canton, thus complying with the norms established by scientific research in the construction of this type of study. Results. An interpretation of the results of the surveys is carried out, considering that the most important data are those that provide information on the frequency and quantity of consumption of the product under study, by the population, which is key for determining the unsatisfied demand. Conclusion. It was determined that there is an unsatisfied demand of $11,968.01 \mathrm{Kg}$ of bee honey, that $52 \%$ of the bee honey consumed in the Macas canton comes from the informal sector, that is to say that there is no quality control on this product, which There is a deeprooted consumption of this product among its inhabitants since $73 \%$ of those surveyed responded that they consume it regularly, which undoubtedly favors the possibility of growth of these productive systems.

Keywords: Market, Honey, Demand, Supply, Unsatisfied Demand

\section{Resumen.}

Introducción. La miel de abeja es un producto milenario que ha sido parte de la alimentación humano y demás usos terapéuticos, está constituida de azucares (fructosa, glucosa, sacarosa), aminoácidos y minerales lo que le ha convertido en un alimento apetecible por el hombre y algunos animales silvestres; a lo largo de la evolución humana se han buscado diferentes formas de aprovechar eficientemente este recurso, que hoy por hoy se ha convertido en una verdadera oportunidad de negocio especialmente en el sector rural espacio que presta las facilidades ecológicas y donde las fuentes de trabajo y subsistencia cada día están más escasas. Objetivo. En este contexto el objetico del presente estudio fue determinar la demanda insatisfecha de miel de abeja en el cantón Morona provincia de Morona Santiago, el documento consta de, este objetivo trata de responder al planteamiento del problema. Metodología. Parte de un enfoque cualitativo y cuantitativo, basado en el método inductivo y deductivo, la muestra poblacional fue de 372 hogares del cantón Morona, cumpliendo así con las normas que establece la investigación científica en la construcción de este tipo de estudios. Resultados. Se realiza una interpretación de los resultados de las encuestas, considerando que los datos más importantes son aquellos que aportan con información sobre la frecuencia y cantidad de consumo del producto en estudio, por parte de la población, lo que significo clave para la determinación de la demanda insatisfecha. Conclusión. Se determino que existe una demanda insatisfecha de $11.968,01 \mathrm{Kg}$ de miel de abeja, que el 52\% de la miel de abeja que se consume en el cantón macas proviene del sector informal es decir que no existe control de calidad sobre este producto, que existe un consumo arraigado de este producto entre sus pobladores ya que el $73 \%$ de los encuestados respondió que lo consume regularmente lo que sin duda favorece la posibilidad de crecimiento de estos sistemas productivos.

Palabras claves: Mercado, Miel de abeja, Demanda, Oferta, Demanda Insatisfecha, 


\section{Introducción.}

La miel es una sustancia producida por las abejas a partir de la transformación del néctar que es recogido en la naturaleza, para alimentación de los miembros de la colmena, sin embargo, desde tiempos inmemorable el ser humano se ha beneficiado como parte de su alimentación debido a sus innumerables beneficios nutricionales e incluso medicinales.

A partir de estas experiencias nace como una actividad productiva y ciencia lo que hoy conocemos como la Apicultura, que según (Hernández, 2017) manifiesta, que esta ciencia se dedica al cultivo de abejas, una tradición milenaria que consiste en cuidar un enjambre de abejas dentro de colmenas, obteniendo de ellas como excedente, la miel, propóleos, polen, jalea real y productos terapéuticos que se utilizan en el tratamiento de algunas de las enfermedades del ser humano.

Para Matamoros y Montano (2018), la producción apícola es una actividad que produce importantes beneficios a la producción agrícola y forestal mediante la acción polinizadora de las abejas, contribuyendo a aumentar la productividad del sistema de explotación y acrecentando la diversidad biológica.

Según Ocaña (2008), referida por Tamayo (2015), la apicultura en el ecuador se inicia aproximadamente en 1870 con la llegada de las primeras colmenas traídas por los hermanos cristianos desde Francia hacia la ciudad de Cuenca, lugar que se convirtió en el principal centro de desarrollo de esta actividad, sin embargo a partir de 1940 esta actividad empieza a llamar la atención de pequeños productores rurales y empieza su verdadero expansión hacia las demás regiones del callejón interandino e incluso hasta zonas costeras, a tal punto que en 1974 de parte del gobierno se crea el Programa de Apicultura a cargo de la Dirección de Desarrollo Campesino, entidad Adscrita al Ministerio de Agricultura y Ganadería (MAG).

Según el Ministerio de Agricultura y ganadería (2018), en el Ecuador actualmente existen inventariados 1760 productores de miel que tienen registradas en producción 19155 colmenas distribuidas de en todo el país de las siguiente manera: Loja con 325 apicultores registrados y 2429 colmenas registradas; Manabí con 146 apicultores registrados y 1820 colmenas registradas; Santa Elena cuenta con 144 apicultores y 828 colmenas; Azuay tiene 118 apicultores y 854 colmenas registradas, mientras que en Chimborazo hay 1190 colmenas registradas pertenecientes a 114 apicultores.

De acuerdo a este registro la actividad apícola va en aumento en el país para la revista vistazo, el rendimiento promedio nacional de miel por colmena se ha incrementado en los últimos tres años de 10,5 a 15,5 kg (Armijos S, 2021).

Pese a estos esfuerzos la producción de miel en el cantón morona Provincia de Morona Santiago, lugar donde realizaremos el presente estudio es un actividad que se lo maneja de forma empírica, cuyos niveles de producción distan mucho del promedio nacional, factor que se agrava, por no existir estudios que determinen la demanda de este producto, y así alcanzar precios justos para los pequeños productores, lo que sin duda desmotiva e 
impide que esta actividad se convierta en una alternativa productiva sostenible para las familias rurales de este sector del país.

\section{El Problema.}

Como hemos manifestado la producción de miel en el cantón Morona es marginal si comparamos con la producción de otras provincias del ecuador, Según el Ministerio de Agricultura y Ganadería, (2018) en la provincia de Morona Santiago existen 24 apicultores, que engloban la producción de 304 colmenas, esto se debe a algunos factores tales como el desconocimiento de las bondades de estos sistemas productivos, poco interés que tienen los pequeños productores porque su producto no alcanza precios competitivos debido a lo que cuesta producir, según estudios de la FAO (2000), el costo de producción de este producto tiene el siguiente comportamiento: Ecuador $\$ 9$, Argentina \$ 4 y México \$5. Finalmente, otro aspecto a consideras y que agrava la situación es que no se dispone de un estudio que permita identificar la demanda que facilite su comercialización.

Sin duda estas circunstancias han determinado que exista poco interés de parte de los productores rurales, pese a las bondades ecológicas que presta el cantón para este tipo de explotación productiva.

\section{Objetivo.}

Realizar un análisis de mercado que permita determinar la demanda insatisfecha de miel de abeja en el cantón Morona.

\section{Fase teórica.}

\section{Mercado.}

Para Córdova M. (2011) mercado es el punto de encuentro de oferentes con demandantes de un bien o servicio, es un espacio físico donde vendedores y compradores llegan a acuerdos sobre calidad, cantidad y precio de un bien o servicio.

\section{Estudio de mercado}

Para Córdova M. (2011) el estudio de mercado busca estimar las cantidades de bienes o servicios que la comunidad adquirirá en un tiempo determinado.

Vaca Urbina (2010) el estudio de mercado nos permite cuantificar la demanda y la oferta, y realizar un análisis de precios y de los mecanismos de comercialización.

Esto sin duda se consigue porque a través de esta herramienta podemos determinar la demanda insatisfecha que resulta de la diferencia de la demanda frente a la oferta de bienes o servicios, adicional a estos criterios el estudio nos permite realizar un análisis de precios de nuestros productos a través de análisis comparativo entre los productos directos y sustitutos.

Objetivos del estudio de mercado. 
Córdova M. (2011) manifiesta que el objetivo del estudio de mercado es

- Verificar que existe un mercado insatisfecho y que es viable, desde el punto de vista operativo, introducir en ese mercado el producto objeto de estudio.

- Demostrar que tecnológicamente es posible producirlo, una vez que se verificó que no existe impedimento alguno en el abastecimiento de todos los insumos necesarios para su producción.

- Demostrar que es económicamente rentable llevar a cabo su realización.

Entre las principales ventajas que nos determina un estudio de mercado es que no permite identificar oportunidades comerciales, a través de la satisfacción de necesidades de la población lo que genera para la empresa crecimiento y sostenibilidad.

Pero como existe ventajas también hay desventajas y la más importante desde el punto de vista económico se relaciona con aquellas empresas donde tienen dentro de su portafolio de ventas varios productos.

Al final del análisis podemos manifestar que un estudio de mercado es mucho más beneficioso que simplemente lanzarse a la aventura de tratar de construir un negocio sobre supuestos porque a la corta o a la larga esto le resultara mucho más costoso.

\section{Identificación del producto.}

Córdova M (2011) manifiesta que el producto es la generación de un bien para satisfacer una necesidad o deseo, sea este supone objetos físicos, servicios, personas, lugares, organizaciones, e ideas; y se clasifica en:

- Bienes no duraderos o fungibles, como los alimentos.

- Bines duraderos, que son aquellos que sobreviven a varios usos como los electrodomésticos.

- Servicios, que son actividades, beneficios o satisfacciones que se ofrecen a la venta.

- Bienes de consumo, que son producidos para ser consumidos por las personas o familias.

- Bienes de capital o de inversión que son utilizados como medios de transformación de la producción de otros bienes o servicios.

De esta clasificación podemos deducir que un bien es todo objeto tangible o intangible que a través de un proceso de transformación generara un beneficio a su posesionario.

\section{El cliente}

Córdova M (2011) define al cliente como el componente fundamental del mercado. Se le denomina "Mercado meta ", ya que será el consumidor del producto o servicio que se ofrecerá con el proyecto, constituyéndose en su razón de ser.

\section{Demanda.}


Para Vaca Urbina G (2010) la demanda es la cantidad de bienes o servicios que el mercado requiere para satisfacer una necesidad específica a un precio establecido, para su determinación se emplean herramientas de la investigación de mercados basadas en la investigación estadística y de campo, en este contexto se entiende por demanda al llamado Consumo nacional aparente (CNA), que es la cantidad de un bien o servicio que el mercado requiere y se puede expresar como:

- $\mathrm{CNA}=$ Producción nacional + las exportaciones - importaciones.

En este sentido cunado se dispone de datos estadístico resulta fácil el poder calcular la demanda, sin embargo, uno de los grandes déficits que tiene nuestro país es que lamentablemente no se generan bases de datos con información estadística útil que esté disponible.

Córdova M (2011), define a la demanda como la cantidad y calidad de bienes y servicios que pueden ser adquiridos a los diferentes precios del mercado por un consumidor (demanda individual) o por el conjunto de consumidores (demanda total o de mercado), en un momento determinado. Y lo clasifica en:

- Demanda agregada: Se refiere al total del gasto en bienes y servicios de una economía en un determinado período de tiempo.

- Demanda derivada: la que es consecuencia de otra demanda. Así, la demanda de capitales y de mano de obra depende de la demanda final de bienes y servicios.

- Demanda elástica: característica que tienen aquellos bienes cuya demanda se modifica de forma sustancial como consecuencia de cambios en el precio de dicho bien o cambios en la renta de los consumidores.

- Demanda inelástica: demanda que se caracteriza porque la variación en el precio de un bien determinado apenas afecta a la variación de la cantidad demandada de ese bien, de forma que queda manifiesta la rigidez de su demanda. En ocasiones esta relación es incluso inexistente, y entonces se habla de total rigidez de la demanda.

- Demanda exterior: demanda de un país de bienes o servicios producidos en el extranjero.

- Demanda interna: suma del consumo privado y del consumo público de bienes y servicios producidos en el propio país.

- Demanda monetaria: función que expresa la cantidad de riqueza que las personas y las empresas guardan en forma de dinero, renunciando así a gastarlo en bienes y servicios o a invertirlo en otros activos.

- Demanda individual: refleja el comportamiento de un individuo o unidad consumidora en particular.

- Demanda de mercado: refleja las preferencias del conjunto de individuos o unidades consumidoras respecto a un determinado bien o servicio. La demanda de mercado se obtiene de agregar para cada precio las cantidades requeridas que cada una de las unidades consumidoras demandaría. 


\section{Metodos de estimación de la demanda}

Vaca Urbina G (2010) manifiesta que la forma más usada en la determinación de la demanda es el método de tendencia secular que se lo puede calcular cuando la demanda y la oferta tiene poca variación en largos periodos de tiempo y su representación gráfica es una línea recta o una curva, y considera 3 métodos:

- Medias Móviles: Consiste en suavizar la variación de las tendencias por medios parciales.

- Mínimos Cuadrados: Este método consiste en calcular una la ecuación de una curva para una serie de puntos dispersos sobre una gráfica, curva que se considera el mejor ajuste, el cual se da cuando la suma algebraica de las desviaciones de los valores individuales respecto a la media es cero y cuando la suma del cuadrado de las desviaciones de los puntos individuales respecto a la media es mínima, su expresión matemática es:

$\mathrm{y}=\mathrm{a}+\mathrm{bX}$ de donde

$$
b=\frac{\sum x y-n \overline{x y}}{\sum x^{2}-n \bar{x}^{2}} \quad a=\bar{y}+b \bar{x}
$$

- Ecuaciones no lineales: Se utiliza Cuando la tendencia del fenómeno en estudio es claramente no lineal, se utilizan ecuaciones que se adapten al fenómeno. Los principales tipos de ecuaciones no lineales son: la parabólica, definida por una ecuación clásica de parábola

$$
\begin{aligned}
& \text { Parábola } Y=a+b X+c X^{2} \\
& \text { Exponencial } Y=a b^{X}
\end{aligned}
$$

Existen además otros métodos del cálculo de la demanda y se basa en la recolección de datos primarios a través de encuestas, cuya información nos determina la frecuencia y tendencia de consumo y a través del consumo percapita y la determinación del mercado potencial podemos calcular la demanda actual esta metodología responde a la siguiente expresión matemática:

$\mathbf{Q}=\mathbf{n} * \mathbf{q}$ de donde

- $\mathrm{Q}=$ demanda actual

- $\mathrm{n}=$ mercado potencial

- $\mathrm{q}=$ consumo percapita

\section{Demanda insatisfecha}


Se llama Demanda Insatisfecha a aquella Demanda que no ha sido cubierta en el Mercado y que pueda ser cubierta, al menos en parte, por el Proyecto; dicho de otro modo, existe Demanda insatisfecha cuando la Demanda es mayor que la Oferta resulta de la diferencia de la demanda calculada menos la oferta (Vásquez L, 2011)

\section{La oferta.}

Según Vaca Urbina G (2010) la oferta es la cantidad de bienes o servicios que un cierto número de oferentes, pone a disposición del mercado a un cierto precio.

Según Córdova M (2011) "la oferta es una relación que muestra las cantidades de una mercancía que los vendedores estarían dispuestos a ofrecer para cada precio disponible durante un periodo de tiempo dado si todo lo demás permanece constante"

La oferta en economía es el conjunto de bienes y/o servicios que están dispuesto para ser vendido en el mercado, en determinado momento y precios concretos. En otras palabras, se puede decir que la oferta está dada por los productos y servicios disponibles para el consumidor (Riquelme, 2021).

\section{Ley de la oferta.}

Es una ley económica por la cual se determina que la cantidad ofrecida de un determinado producto o servicio aumenta a medida que aumenta su precio, mientras que las restantes variables se mantienen constantes. Es decir, la ley de la oferta determina la cantidad ofertada de un bien en dependencia de su precio, señalando que, a mayor precio de un bien, mayor será la oferta, ya que los productores se sienten motivados a colocar sus productos en el mercado (Riquelme, 2021).

\section{Elasticidad de la oferta.}

Se designa como elasticidad de la curva de oferta, la variación porcentual que experimenta la cantidad ofertada de un determinado bien, cuando su precio varía solo en $1 \%$, conservándose constantes los otros factores que pudiesen afectar la cantidad ofertada (Riquelme, 2021).

\section{Elasticidad de la relación precio - oferta}

Córdova M (2011), muestra el cambio porcentual en la cantidad ofrecida que resulta de un cambio porcentual en el precio. Busca medir el impacto en la oferta de un producto o servicio dada una variación en su precio, así:

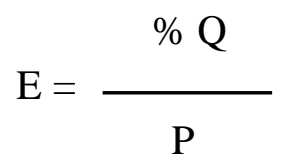

\section{Principales tipos de oferta}


- Oferta competitiva o de mercado libre: En ella los productores se encuentran en circunstancias de libre competencia, sobre todo debido a que existe tal cantidad de productores del mismo artículo, que la participación en el mercado está determinada por la calidad, el precio y el servicio que se ofrecen al consumidor

- Oferta oligopólica (del griego oligos, poco): Se caracteriza porque el mercado se encuentra dominado por sólo unos cuantos productores.

- Oferta monopólica: Es en la que existe un solo productor del bien o servicio y, por tal motivo, domina por completo el mercado e impone calidad, precio y cantidad. Un monopolista no es necesariamente un productor único. Si el productor domina o posee más de $95 \%$ del mercado siempre impondrá precio y calidad (Vaca Urvina G, 2010).

En terminaos generales el estudio de la oferta es el estudio de la competencia ya sea de productos directos o sustitutos.

\section{Precio.}

El precio desde el punto de vista conceptual es el valor que se le pone a un bien o servicio expresado en valor monetario, es decir que el precio señala la cantidad de dinero que un demandante de un bien o servicio pagara por poseer dicho bien.

Para Córdova M (2011) El precio es el regulador entre la oferta y la demanda, salvo cuando existe protección (aranceles, impuestos). El estudio de precios tiene gran importancia e incidencia en el estudio de mercado, ya que de la fijación del precio y de sus posibles variaciones dependerá el éxito del producto o servicio a ofrecer.

Vaca Uribe G (2010) define al precio como la cantidad monetaria a la cual los productores están dispuestos a vender y los consumidores a comprar un bien o servicio, cuando la oferta y la demanda están en equilibrio.

\section{Objetivos del analisis de precios.}

Córdova (2011) se plantea los siguientes objetivos:

- La determinación de cambios basada en cifras indicadoras.

- Determinación e interpretación de la elasticidad del precio.

- Investigación de los precios de la competencia: catálogo, descuento, márgenes, tendencia.

Tipos de precios.

Vaca Urbina G. (2010) considera la existencia de los siguientes tipos de precios:

- Internacional: Es el que se usa para artículos de importación-exportación. Normalmente está cotizado en dólares estadounidenses y FOB (libre a bordo) en el país de origen.

- Regional externo: Es el precio vigente sólo en parte de un continente. Por ejemplo, Centroamérica en América; Europa occidental en Europa, etc. Rige para 
acuerdos de intercambio económico sólo en esos países, y el precio cambia si sale de esa región.

- Regional interno. Es el precio vigente en sólo una parte del país. Por ejemplo, en el sureste o en la zona norte. Rigen normalmente para artículos que se producen y consumen en esa región; si se desea consumir en otra, el precio cambia.

- Local: Precio vigente en una población o poblaciones pequeñas y cercanas. Fuera de esa lo-calidad el precio cambia.

\section{Criterios para la definición de precios.}

En general el precio depende de algunos criterios entre los que podemos destacar:

- Elasticidad de la demanda y la oferta.

- Precio del producto directo o sustituto de la competencia

- El posicionamiento y fidelización del producto en el mercado.

- De las políticas gubernamentales en la fijación de precios oficiales.

- Del segmento de mercado al que esté orientado mi producto y canales de distribución.

- Estructura de los costos de producción

- Rentabilidad esperada de la venta del producto

- Calidad de la materia prima utilizada.

\section{Comercilización}

\section{Distribución.}

Uno de los aspectos más importantes que se debe considerar en la venta de un bien o servicio es la forma como llegaremos a nuestros clientes, esta puede ser de dos tipos:

- Venta Directa: es decir que la empresa se dedicara a la distribución directa a los consumidores finales del bien o servicio a través de la implementación de puntos de venta. Esto sin duda requiere de un profundo análisis en donde debe primar el criterio de beneficio costo porque establecer la estrategia de venta directa significara una mayor inversión para la empresa y por tanto un mayor riesgo.

- Venta Indirecta: Significa que la empresa va a vender sus productos a través de distribuidores, al igual que en el caso anterior la decisión está en función del beneficio costo y de los ventajas y desventajas que este sistema de distribución me ofrece.

En cuanto al transporte de la mercadería también se debe analizar que más le conviene a la empresa si utilizar un sistema de transporte propio y simplemente contratar el servicio en todo caso como en toda actividad económica el criterio que permitirá decidir el sistema a utilizar siempre estará en función del beneficio costo.

Uno de los aspectos importantes dentro de los sistemas productivos es el análisis del aprovisionamiento de materia prima y demás productos indirectos de fabricación, que sin duda es un factor determinante en la construcción del costo de producción, es por eso que 
al momento de seleccionar a los proveedores estos deben ofrecer ventajas significativas, que sin duda tienen una relación directa con su costo.

\section{Estrategias de introducción al mercado.}

Se refiriere a las estrategias que la empresa utilizara para introducir el producto al mercado y que sea aceptado rápidamente por los consumidores. Sin duda esto es de vital importancia ya que de esto dependerá la permanencia o no de nuestro producto en el mercado.

Para Tomas D. (2020). Una estrategia de marketing es el proceso que permite que una empresa se centre en los recursos disponibles y los utilice de la mejor manera posible para incrementar las ventas y obtener ventajas respecto de la competencia. Las estrategias de marketing parten de la base de los objetivos de negocio de la empresa. Para poder aplicar estos objetivos a la elaboración de un plan, necesitamos concretarlos y definirlos mediante el famoso acrónimo "SMART"

- Specific (específicos): un objetivo debe referirse a un logro concreto y tangible.

- Measurable (medibles): tenemos que tener claro qué herramientas de medición se van a emplear para saber si el objetivo se ha conseguido o no.

- Achievable (alcanzables): es fundamental que los objetivos presenten un reto realista, ya que de lo contrario solo conseguiremos descuadrar las predicciones y desmotivar al equipo.

- Relevant (relevantes): los objetivos deben estar relacionados con las metas globales de la empresa y mostrar una correlación clara. Por ejemplo, si queremos conseguir un impacto en las ventas, la métrica a seguir debería ser el número de conversiones y no las interacciones en Facebook.

- Time-bound (con fecha límite): el horizonte temporal de la estrategia de marketing tiene que estar claramente definido desde un principio.

Otro aspecto fundamental de la estrategia de marketing es lo que se conoce como marketing mix o "las $4 \mathrm{P}$ del marketing", que definen cuatro aspectos clave para los planes de empresa:

- El producto: o servicio: lo que nuestra empresa aporta a los clientes. Aquí se incluye la cartera de productos y servicios de la empresa, cuál es su ciclo de vida, cómo se diferencian de la competencia, el branding y el packaging, entre otros aspectos. Hay que tener en cuenta que la definición del producto debe partir de las necesidades y motivaciones del cliente antes que de los aspectos técnicos.

- El precio: el precio de los productos es una de las decisiones de marketing más complejas, ya que intervienen múltiples factores como los costes de fabricación, el margen comercial, la demanda, el posicionamiento respecto a la competencia, el poder adquisitivo de nuestro público potencial y un largo etcétera. En este apartado también se contemplan aspectos como la modalidad de pago o los descuentos. 
- La promoción: todas las acciones de comunicación que la empresa lleva a cabo para dar a conocer los productos y servicios a fin de aumentar las ventas. Incluye diferentes ramas de la comunicación, como la publicidad, las relaciones públicas, la venta puerta a puerta o el marketing digital.

- El punto de venta o distribución: los diferentes canales a través de los cuales los productos y servicios llegan a los consumidores, desde las tiendas online hasta las cadenas comerciales. Este punto abarca aspectos como el acceso, el almacenamiento, la gestión de transporte o los inventarios, además de la experiencia de cliente.

En general un estudio de mercado aporta con información valiosa sobre la oportunidad de mercado que tiene mi producto, y me da la referencia del tamaño de inversión que debo realizar en mi proyecto, pero es importante mencionar también que como hemos señalado anteriormente el hecho de determinar que existe una demanda insatisfecha de mi producto, no significa que este se va vender solo, por tanto es indispensable a este proceso acompañarle con un estudio de investigación de mercados cuales son las características que debe cumplir mi producto para que este tenga una mayor posibilidad de ser aceptado por los consumidores.

\section{Definciones sobre el producto.}

\section{Miel de abeja}

Se definida como una sustancia dulce, no fermentada, producida por las abejas (Apis mellifera) que recogen y procesan el néctar de las flores o de las secreciones de ciertas especies de plantas. Las abejas, transforman y combinan esta sustancia con otras específicas propias que finalmente almacenan y maduran en panales (Schencke et al., 2016)

la FAO (2000), manifiesta que El aroma, gusto y color de la miel son determinados por las plantas de las cuales las abejas han recogido el néctar. Los girasoles, por ejemplo, le dan un color dorado; el trébol produce una miel blanca y dulce; las agaves le dan a la miel un sabor desabrido, muy popular en algunas sociedades. La miel oscura generalmente tiene un sabor fuerte y a menudo presenta un alto contenido mineral; la miel clara tiene un sabor más delicado. La popularidad de las mieles oscuras y claras cambia de país a país. El color puede ser también sinónimo de calidad, porque la miel se vuelve más oscura durante su almacenamiento si es climatizado. Sin embargo, algunos tipos perfectamente frescos y no climatizados pueden ser de color oscuro.

\section{Valor de la miel de abeja.}

la FAO (2000) Considera que la miel tiene un amplio valor entre los que se destacan los siguientes

- Como alimento: La miel es apreciada en todas partes como un alimento dulce y apetitoso. En períodos de escasez es una fuente útil de carbohidratos que contiene oligoelementos y agrega una diversidad nutritiva en un régimen alimenticio 
demasiado pobre. La miel ocupa un lugar importante en la preparación de alimentos tradicionales.

- Como medicina o jarabe: En muchas partes del mundo la miel es utilizada como medicina o jarabe y como tratamiento especial para niños. La medicina moderna está aumentando el uso de la miel en una gran variedad de tratamientos.

- Como producto de valor comercial: La miel fresca local es siempre considerada de mejor calidad que la miel de importación. Muchos apicultores venden sus productos directamente a los consumidores. La miel es usada con frecuencia como un bien de intercambio en algunas aldeas, especialmente en áreas remotas o aisladas por la guerra o bloqueos económicos. La miel es un bien estable que se conserva por mucho tiempo. Si es cosechada cuidadosamente puede ser conservada en cualquier lugar por varios años.

- Como producto de exportación: Con el mejoramiento de los niveles de vida, aumenta el consumo de miel. Muchos países industrializados importan miel para satisfacer sus demandas. Esto significa una fuente importante de divisas para los países en vías de desarrollo exportadores de miel. Los mayores exportadores de miel son México, China y Argentina. Cada uno de estos países tiene una gran industria de apicultura, que tiene un papel importante en su economía agrícola. (Vea Tabla 1). Todos los países en vías de desarrollo pueden exportar la miel si la producción excede sus demandas internas. Ya que la apicultura no utiliza terrenos, la producción de miel para la exportación no entra en conflicto con la cosecha de otros productos para el consumo interno.

- Como alimento cultural: La miel es usada ampliamente como una fuente de azúcares para producir vino y cerveza de miel. Tiene también un alto valor cultural: consumir miel o usarla para ungüentos forman parte de muchas ceremonias tradicionales, nacimientos, casamientos, funerales, etc. Esta conexión cultural es evidente en la "luna de miel". En las tribus africanas Masai, la miel es usada para pagar el valor de la esposa, en Etiopía el vino de miel es ofrecido en los matrimonios.

- Como agentes polinizadoras: La polinización es fundamental para que las plantas en flor produzcan cualquier tipo de semilla y de frutas. El intercambio de polen entre las flores, tiene el objetivo de la reproducción, es un proceso fundamental para el mantenimiento de la vida sobre la tierra. Las abejas melíferas son insectos polinizadores altamente eficaces:

- Tienen el cuerpo cubierto de pelos que recogen fácilmente miles de gránulos de polen cuando se mueven al interno de las flores.

- Visitan solamente una especie de flor durante cada uno de sus viajes.

- Cada abeja recoge la cantidad suficiente de polen para su propio alimento y también para las necesidades de la colonia. En una sola jornada una abeja puede visitar miles de flores de una misma especie, recogiendo el néctar y el polen y esparciendo interminablemente los gránulos de polen por todas las flores. 
- Propiedades de la miel.

Tabla 1

Propiedades Organolépticas de la miel de abeja DESCRIPCIÓN

Está relacionado con un fenómeno que se conoce como Consistencia cristalización de azucares sobresaturados que posee la miel en su composición en mayor o menor cantidad, estos azucares cristalizados establecen el grado fluidez total o parcial

Existe una escala de colores que oscila entre el blanco agua hasta mieles oscuras de tonos casi negras. Se identifican algunos colores intermedios como tonos ámbar, rojizas, grisáceas y verdosas

Color depende de la cantidad de algunos componentes como fosfato de calcio y hierro o de la porción vitamínica existente. En mieles de tono claro muestran altas concentraciones de vitamina A en contraste con las más oscuras en donde la cantidad de vitamina B y $\mathrm{C}$ es mayor.

Está conjugado a componentes propios dentro de los que se

Sabor destacan los polifenoles y aminoácidos. Depende también de compuestos no volátiles o compuestos que suscitan el dulzor nativo de los diferentes procesos de extracción de miel.

Se afilia a la amplia variedad de flora o especies vegetales que

Aroma agradan a cada tipo de abeja originando gran cantidad de aromas específicos provenientes de cada fuente de néctar

Fuente: (Gutiérrez D, 2016).

Tabla 2

Propiedades físicas de la miel de abeja.

CARACTERÍSTICA DESCRIPCIÓN

Viscosidad

En la miel está relacionada con la temperatura, $1 \%$ de humedad es equivalente a $3,5^{\circ} \mathrm{C}$.

La humedad es una de las características que influye en el peso

Humedad específico, en la viscosidad, en el sabor y condiciona por ello la conservación, la palatabilidad y la solubilidad de la miel, oscila entre el $13-25 \%$ dependiendo de las condiciones climáticas.

Conductividad

eléctrica

La conductividad eléctrica de la miel varía entre los 0.868 a los

$3.65 \times 10-4 \mathrm{ohm} / \mathrm{cm}$. Estos valores aumentan según el contenido de cenizas.

Se produce más rápidamente cuanto más elevada es la relación

Cristalización glucosa/agua. Generalmente esta relación oscila entre 1.6 y 2.5 de viscosidad.

Tabla 2

Propiedades físicas de la miel de abeja. (continuación)

\section{CARACTERÍSTICA DESCRIPCIÓN}


Coloración

El color de la miel va de blanco al negro. Se aprecia por medio Fuente: (Gutiérrez D, 2016). especie percoreada y la rapidez de la secreción.

\begin{tabular}{ll} 
& \multicolumn{1}{c}{$\begin{array}{c}\text { Tabla } 3 \\
\text { Propiedades químicas de la miel. }\end{array}$} \\
\hline CARACTERÍSTICA & \multicolumn{1}{c}{ DESCRIPCIÓN } \\
\hline $\mathrm{pH}$ & $\begin{array}{l}\text { El pH de la miel está comprendido generalmente entre 3.3 y } 4.9 \\
\text { dependiendo del origen de la miel. }\end{array}$ \\
& $\begin{array}{l}\text { El ácido principal de la miel es el ácido glucónico, que proviene } \\
\text { de la oxidación de la glucosa. La cantidad de ácido glucónico } \\
\text { depende principalmente del tiempo transcurrido entre la toma } \\
\text { del néctar por las abejas. }\end{array}$ \\
Acidez & $\begin{array}{l}\text { El contenido de cenizas es un criterio para evaluar el origen } \\
\text { botánico de la miel de abejas. Las mieles florales tienen en } \\
\text { promedio de 0.17\% de cenizas mientras que no florales } \\
\text { presentan un valor más elevado. } \\
\text { Minerales (cenizas) } \\
\text { Los azúcares representan del 95\% al 99\% de la materia seca de } \\
\text { la miel (80-82\% del total), están relacionados con el origen } \\
\text { botánico. La glucosa y fructosa constituyen el 85-90 \% de los } \\
\text { azúcares totales. }\end{array}$ \\
Azúcares totales
\end{tabular}

Fuente: (Gutiérrez D, 2016).

\section{Metodologia.}

\section{Enfoque de la investigación.}

Utilizaremos un enfoque cualitativo ya que este busca comprender la realidad que se investiga en el caso del presente trabajo lo que tratamos es de comprender e interpretar la realidad del mercado de la miel.

\section{Tipos de investigación}

En el presente trabajo utilizaremos la investigación descriptiva, porque al ser un estudio de mercado este se basa en la información u opiniones de personas recogidas a través de cuestionarios estructurados que nos ayudaran a interpretar la información

\section{Metodos, tecnicas e instrumentos.}

El presente trabajo investigativo requiere la participación de grupos sociales quienes a través de la información que proporcionan mediante encuestas permitirán la realización e interpretación de su testimonio, contribuyendo al cumplimiento de los objetivos planteados.

\section{Métodos.}


- Inductivo. Nos permite concluir de manera general sobre la base de información que explica dichos fenómenos.

- Deductivo. Consiste en concluir sobre la sobre las existencias de premisas previas (Encuestas)

- Analítico. Proporciona la oportunidad de analizar la información sobre las tendencias de consumo de la miel de abeja en el cantón Morona, esta información será recogida a través de encuestas.

\section{Técnicas.}

- Observación. Nos proporciona información directa sobre las necesidades de la población sobre temas específicos, inclusive permite la observación de tendencias de consumo

- Entrevista. Recaba información de manera verbal. Esta técnica se utilizará en nuestro trabajo investigativo especialmente en población que por su grado de escolaridad le es muy difícil escribir.

- Encuesta. Es una técnica universalmente utilizada para generar información primaria de distinta clase y población, en este caso realizaremos encuestas a los pobladores completamente al azar para evitar sesgo en información recabada.

\section{Instrumentos.}

Se utilizará cuestionarios (preguntas) orientadas a recabar información que determinen las tendencias de consumo de tal forma que podamos contar con la información necesaria para analizarla y generar las conclusiones que se generen.

\section{Población y muestra.}

\section{Población.}

Se llama población al conjunto de individuos que habitan un mismos entorno geográfico y ecológico y que comparten ciertas características lo que les hace diferentes a la población de otro lugar.

Para Nuestro estudio la población donde se realizará la investigación es el cantón macas, de acuerdo a datos del INEC (s/f) la población proyecta al 2020 el número de habitantes del cantón Morona es de 58.280 mediante el método de mínimos cuadrados se proyectó el número de pobladores al 2021 estimándose que para este año la población creció a 60.385 personas siendo este el universo sobre el que realizaremos nuestro estudio de mercado.

\section{Muestra.}

Dentro de la teoría de muestre se considera la muestra como una parte que representa a una población cuyo principio es que todos sus individuos comparten características 
comunes a las de la población original, de tal forma que la información que genere este grupo de individuos se puede transferir al de la población.

Para nuestro estudio al ser la miel un producto que no se consume de forma individual segmentaremos por hogar considerando la información reportada por el INEC (s/f) que señala que el número de integrantes por hogar para el cantón Morona es de 4,12 personas valor que nos sirve para estratificar nuestra muestra a número de hogares resultando que son 12.007 hogares que serán parte de nuestro estudio. En base a estos criterios procederemos a calcular el tamaño de la muestra. Con la siguiente expresión matemática.

$$
n=\frac{N \sigma^{2} Z^{2}}{(N-1) e^{2}+\sigma^{2} Z^{2}}
$$

De donde:

- $\mathrm{n}=$ Tamaño de la muestra

- $\mathrm{N}=$ Tamaño de la población que para nuestro estudio es de 11.656 familias

- $\partial=$ Desviación estándar de la población que, generalmente cuando no se tiene su valor, suele utilizarse un valor constante de 0,5 .

- $\mathrm{Z}=$ Valor obtenido mediante niveles de confianza. Es un valor constante que, si no se tiene su valor, se lo toma en relación al 95\% de confianza equivale a 1,96 .

- Límite aceptable de error maestral que, generalmente cuando no se tiene su valor, suele utilizarse un valor que varía entre el $1 \%(0,01)$ y $9 \%(0,09)$, valor que queda a criterio del encuestador.

$$
\begin{aligned}
& n=\frac{12.007 * 0.5^{2} * 1.96^{2}}{(11656-1) * 0.05^{2}+0.5^{2} * 1.96^{2}} \\
& n=372
\end{aligned}
$$

De acuerdo a los resultados obtenidos para nuestro estudio debemos realizar 372 encuestas en forma aleatoria a familias del cantón morona, esta actividad nos permitirá recabar información sobre las tendencias de consumo de miel de abeja de los hogares del cantón Morona.

En cuanto a la herramienta (encuesta) se desarrollará preguntas que, a más de generar información de la frecuencia y cantidad de consumo, también sirva de base para determinar cuál es la opinión de las personas con respecto a: los precios, presentación, y preferencias de consumo. 


\section{Resultados.}

\section{Procesamiento y presentación de resultados}

En la siguiente tabla se presente información sobre la opinión a las diferentes preguntas realizadas en las encuestas

\section{Tabla 4}

Presentación de resultados: encuestas realizadas a los pobladores del canton Morona

\section{PREGUNTA}

1. ¿Usted consume miel de abeja?

2. ¿Considera importante el consumo de la miel de abeja dentro de su canasta básica?

3. ¿Con que frecuencia consume usted miel de abeja y en qué cantidad?

4. ¿Qué cantidad de frascos de miel de abeja de 250 gramos consume mensualmente?

5. ¿Cuál es la marca de la miel de abeja que consume?

6. ¿Usted cómo califica al producto que consume?

7. ¿Conoce los beneficios y propiedades que tiene la miel de abeja?

\section{RESULTADO}

El $73 \%$ sí consume miel de abeja y el $27 \%$ no consume este producto.

El $81 \%$ considera que sí es importante el consumo de miel de abeja en la canasta básica, mientras que el 19\% no lo considera necesario.

El $43 \%$ de los consumidores consume una unidad de miel de abeja por mes, el $26 \%$ consume una unidad cada quince días, el $11 \%$ consume una unidad semanalmente y el $20 \%$ de los encuestados manifestó que consume cada tres meses, considerando unidades de 250 gramos.

El 54\% consume un frasco por familia, el $27 \%$ consume de 2 a 3 frascos por familia y el 19\% consume de 4 a 5 frascos mensualmente por familia.

El $77 \%$ consume miel de abeja sin importarle la marca, el $4 \%$ consumen la marca Schullo, el $4 \%$ consume la marca Selva Miel, el $13 \%$ de los encuestados consume la marca Namavit, y el 2\% la marca Productos del Bosque. Sin embargo, un número importante de encuestados manifestaron que consumen León Miel, aun cuando esta no era una opción de la encuesta.

El $40 \%$ de los consumidores califica al producto como muy bueno, el $11 \%$ califica como bueno y un $49 \%$ califica como excelente.

El $72 \%$ de los consumidores conocen los beneficios y propiedades de la miel de abeja, mientras que un $28 \%$ desconocen los mismos.

Fuente: (Leon H, 2021)

\section{Analisis de la demanda de miel de abeja en el canton morona.}

Para Vaca Urbina G (2010) la demanda es la cantidad de bienes y servicios que el mercado requiere o solicita para buscar la satisfacción de una necesidad específica a un precio determinado.

Para calcular la demanda Actual (QA), utilizamos la siguiente expresión matemática:

$$
\mathrm{QA}=\mathrm{n} * \mathrm{Cpc}
$$


De donde:

- $\mathrm{Q}=$ Demanda actual

- $\mathrm{n}=$ Población o mercado meta

- $\mathrm{Cpc}=$ Consumo percapita.

Para la determinación de la demanda actual es necesario calcular la población o merado meta y el consumo percapita, para lo cual nos apoyaremos en los resultados de las encuestas realizadas a la población de macas.

\section{CÁlCULO DEL DE LA POBLACION O MERCADO META (n).}

Para este cálculo nos apoyamos en las repuesta a las encuestas, de la pregunta 1 en la que nos manifiestan que el $73 \%$ de los encuestados consumen miel, de donde:

\section{$\mathrm{n}=$ Población total $*$ personas aue consumen}

De donde:

$$
\mathrm{n}=(12007 \text { hogares }) *(73 \%)=\mathbf{n}=\mathbf{8 8 1 6} \text { hogares }(\text { mercado meta }) .
$$

\section{CALCULO DEL CONSUMO PERCAPITA (q)}

Es el consumo total (de productos, de alimentos, de agua, de energía, etc.) de un país o región dividido por el número de sus habitantes en determinado periodo de tiempo. Es un indicador que permite medir y analizar los índices de consumo en una población (Significados, 2021).

Partiendo de este concepto, para determinar su valor partimos de la pregunta 3 y 4 que nos determina la frecuencia y cantidad consumida de miel por las personas. Para el cálculo del consumo percapita pariremos de la siguiente pregunta ¿Cuántos frascos de miel de 2050gr de miel de abeja consume en su hogar cada mes?

\section{Tabla 5}

Frecuencia y cantidad de consumo de miel de abja por hogar

\begin{tabular}{lccccc}
\hline $\begin{array}{l}\text { Alternativas en } \\
\text { unidades de 250 } \\
\text { gramos }\end{array}$ & Frecuencia & \% & $\begin{array}{c}\text { Marca de } \\
\text { clase }\end{array}$ & Frecuencia & Producto \\
\hline 1 & 170 & 63 & 1 & 170 & 170 \\
$2-3$ & 65 & 24 & 2.5 & 65 & 162.5 \\
$4-5$ & 35 & 13 & 4.5 & 35 & 157.5 \\
$5-6$ & 0 & 0 & 0 & 0 & 0 \\
\hline TOTAL & 270 & 100 & & & 490 \\
\hline
\end{tabular}

Fuente: Investigación de campo

Realizado por: Grupo de investigación (2021).

A partir de estos datos determinamos el consumo percapita:

$$
\mathrm{Cpc}=\frac{490}{270} \rightarrow \mathrm{Cpc}=1,81 \text { frascos de miel } / \text { mes }
$$




\section{$\mathrm{Cpc}=46.204,01 \mathrm{~kg}$ de miel/año}

Cálculo de la demanda actual (qa)

$$
\mathrm{QA}=\mathrm{n} * \mathrm{Cpc}
$$

$\mathrm{QA}=(8.816$ hogares $) *(5,43 \mathrm{Kg}$ de miel/año $)$.

$\mathrm{QA}=47.872,02 \mathrm{Kg} / \mathrm{año}$.

Proyección de la demanda para los siguientes 3 años

Tabla 6

Proyección de la demanda para los proximos 3 años

\begin{tabular}{|c|c|c|c|c|c|c|c|}
\hline Año & Población & $\begin{array}{l}\text { Número de } \\
\text { integrantes } \\
\text { por hogar }\end{array}$ & $\begin{array}{c}\text { Número } \\
\text { de } \\
\text { familias }\end{array}$ & $\begin{array}{l}\text { Porcentaje de } \\
\text { hogares } \\
\text { consumidores } \\
\text { de miel de } \\
\text { abeja }(73 \%)\end{array}$ & $\begin{array}{c}\text { Población } \\
\text { meta } \\
\text { (familias) }\end{array}$ & $\begin{array}{c}\text { Consumo } \\
\text { per- } \\
\text { cápita } \\
\text { (kg/año) }\end{array}$ & $\begin{array}{c}\text { Demanda } \\
\text { (kg/año) }\end{array}$ \\
\hline 2021 & 60385 & 4,12 & 12077 & 0,73 & 8816 & 5,43 & 47872,02 \\
\hline 2022 & 62565 & 4,12 & 12513 & 0,73 & 9134 & 5,43 & 49600,28 \\
\hline 2023 & 64824 & 4,12 & 12965 & 0,73 & 9464 & 5,43 & 51391,17 \\
\hline 2024 & 67164 & 4,12 & 13433 & 0,73 & 9806 & 5,43 & 53246,28 \\
\hline
\end{tabular}

Fuente: Investigación de campo

Realizado por: Grupo de investigación (2021).

\section{Análisis de la oferta de miel de abeja en canton morona.}

Según Vaca Urbina G (2010), la oferta es la cantidad de bienes o servicios que un cierto número de oferentes (productores) está dispuesto a poner a disposición del mercado a un precio determinado. Partiendo de este concepto para la determinación de la oferta de miel de abeja en el cantón morona procedimos a determinar la frecuencia de consumo en los diferentes centros de expendio del cantón, observándose su consumo está en función de la marca, resultados que se sustentan en la pregunta 4 de las encuestas los mismos que presentamos en la tabla 7 .

\section{Tabla 7}

Frecuencia y cantidad de miel ofertada en el cantón Morona.

\begin{tabular}{cccc}
\hline $\begin{array}{c}\text { Demanda } \\
\text { insatisfecha }\end{array}$ & Marca de miel & $\begin{array}{c}\text { Frecuencia de } \\
\text { consumo por marca }\end{array}$ & $\begin{array}{c}\text { Oferta de miel de } \\
\text { abeja en Kg. }\end{array}$ \\
\hline 47872,02 & Namavit & $13 \%$ & 6223,36 \\
47872,02 & Schullo & $4 \%$ & 1914,88 \\
47872,02 & Selva Miel & $4 \%$ & 1914,88 \\
47872,02 & Productos del & $2 \%$ & 957,44 \\
47872,02 & bosque & $52 \%$ & 24893,45 \\
TOTAL, MIEL DE ABEJA OFERTADA & & $\mathbf{3 5 9 0 4 , 0 2}$ \\
\hline
\end{tabular}

Fuente: Investigación de campo

Realizado por: Grupo de investigación (2021) 
De acuerdo a estos resultados se estima que la cantidad ofertada de miel de abeja en el cantón morona es de $35.904,02 \mathrm{Kg}$ de miel que representa el $75 \%$ del total de la miel de abeja demandad en el cantón, observándose que la mayor cantidad de consumo es aquella que se comercializa en la informalidad en decir sin ningún tipo de control sanitario y de calidad.

\section{Proyección de la oferta.}

\section{Tabla 8}

Oferta proyectada de miel de abeja

\begin{tabular}{|c|c|c|c|c|c|c|c|c|c|}
\hline Año & Población & $\begin{array}{c}\text { Número de } \\
\text { integrantes } \\
\text { por hogar }\end{array}$ & $\begin{array}{c}\text { Número } \\
\text { de } \\
\text { familias }\end{array}$ & $\begin{array}{c}\text { Porcentaje de } \\
\text { hogares } \\
\text { consumidores } \\
\text { de miel de } \\
\text { abeja }(73 \%)\end{array}$ & $\begin{array}{c}\text { Población } \\
\text { meta } \\
\text { (hogares) }\end{array}$ & $\begin{array}{l}\text { Consumo } \\
\text { per- } \\
\text { cápita } \\
\text { (kg/año) }\end{array}$ & $\begin{array}{c}\text { Demanda } \\
\text { (kg/año) }\end{array}$ & $\begin{array}{c}\text { Miel } \\
\text { Ofertada } \\
\text { en } \%\end{array}$ & $\begin{array}{c}\text { Oferta } \\
\text { proyecta } \\
\text { de miel }\end{array}$ \\
\hline 2021 & 60385 & 4,12 & 12077 & 0,73 & 8816 & 5,43 & 47872,02 & $75 \%$ & 35904,02 \\
\hline 2022 & 62565 & 4,12 & 12513 & 0,73 & 9134 & 5,43 & 49600,28 & $75 \%$ & 37200,21 \\
\hline 2023 & 64824 & 4,12 & 12965 & 0,73 & 9464 & 5,43 & 51391,17 & $75 \%$ & 38543,38 \\
\hline 2024 & 67164 & 4,12 & 13433 & 0,73 & 9806 & 5,43 & 53246,28 & $75 \%$ & 39934,71 \\
\hline
\end{tabular}

Fuente Investigación de campo

Realizado por: Los autores (2021)

\section{Analisis de la demanda insatisfecha}

La demanda insatisfecha es aquella que demanda que no es cubierta por el mercado se la obtiene de la resta de la demanda menos la oferta. A continuación de detalla el cálculo realizado en nuestro estudio.

\section{Tabla 9}

Cálculo de la demanda insatisfecha de miel de abeja en el cantón Morona para los próximos 3 años

\begin{tabular}{cccc}
\hline Año & $\begin{array}{c}\text { Demanda } \\
\text { proyectada de } \\
\text { miel de abeja }\end{array}$ & $\begin{array}{c}\text { Oferte } \\
\text { proyectada de } \\
\text { miel de abja }\end{array}$ & $\begin{array}{c}\text { Demanda insatisfecha de } \\
\text { miel de abeja }\end{array}$ \\
\hline 2021 & 47872,02 & 35904,02 & 11968,01 \\
2022 & 49600,28 & 37200,21 & 12400,07 \\
2023 & 51391,17 & 38543,38 & 12847,79 \\
2024 & 53246,28 & 39934,71 & 13311,57 \\
\hline
\end{tabular}

Fuente: Investigación de campo

Realizado por: Los autores (2021)

\section{Conclusiones.}

El estudio genera las siguientes conclusiones:

- La mayor cantidad de miel comercializada en el cantón Morona proviene del comercio informal de acuerdo al análisis de datos se pudo determinar que el 52\% 
de este producto se vende sin ningún tipo de control en cuanto a la inocuidad del producto.

- La demanda insatisfecha actual estimada para el cantón de macas es de $11.968,01 \mathrm{Kg}$ de miel para el año 2021 eso significa que existe la posibilidad de crecimiento de este sector, factor que es fortalecido debido a las condiciones medioambientales favorables que ofrece el cantón macas.

- La miel de abeja es un producto que está muy arraigado y presente en la cultura alimenticia de los hogares de macas, ya que el $73 \%$ de las personas encuestadas manifestaron que consumen con frecuencia este producto.

- Se estima que la oferta de miel de abeja cubre el $75 \%$ de la demanda de este producto.

- Del $75 \%$ de miel ofertada en el cantón Morona el 23\% se lo comercializa cumpliendo las normas de sanitarias establecidas para la venta de este producto y no se pudo determinar el origen del mismos, pero asumimos que su casi la totalidad proviene de otros sectores del país

- El presente estudio tomo como referencia en el proceso de segmentación a hogares ya que la miel es un producto de consumo colectivo.

\section{Referencias bibliográficas.}

Córdova, M. (2011). Formulación y evaluación de proyectos. 2da Edición Eco ediciones.

FAO. (2000). La miel un alimento popular. http://www.fao.org/3/y5110s/y5110s05.htm\#bm05.1

Gutiérrez D. (2016). Relación estadística de las propiedades químicas, físicas y microbiológicas de tres muestras de mieles (apis mellifera.) comerciales, distribuidas en supermercados del distrito metropolitano de quito. (Tesis de Pregrado, Universidad Politécnica Salesiana) file:///C:/Users/Usuario/Downloads/UPS-QT11176.pdf

INEC (s/f). Ecuador en cifras 2017. https://www.ecuadorencifras.gob.ec/wpcontent/descargas/Manu-lateral/Resultados-provinciales/morona_santiago.pdf

Matamoros P. y Montano C (2018). Características físico y químico de la miel de abeja en el distrito de Acoria - Huancavelica. (Tesis de Pregrado, Universidad Nacional de

Huancavilca).

https://repositorio.unh.edu.pe/bitstream/handle/UNH/2809/TESIS-2018-

ING.\%20MINAS-

PACO\%20MATAMOROS\%20Y\%20MONTANO\%20CRISOSTOMO.pdf?seq uence $=1 \&$ is Allowed $=\mathrm{y}$

Ministerio de Agricultura y Ganaderia ( 2018). Fomenta la prooducción apícola en Morona Santiago. https://www.agricultura.gob.ec/mag-fomenta-la-apicultura-enmorona-santiago/ 
Ministerio de Agricultura y Ganaderia ( 2018). Registro apícola. Consultado el 2 de septiembre del 2021 https://www.agricultura.gob.ec/ecuador-tiene-1760apicultores-registrados/

Leon H. (2015). Proyecto para la implementación de un centro de acopio y distribución de miel de abeja en el canton Morona. (tesis de Pregrado, Escuela Superior Politécnica de Chimborazo).

Riquelme M. (2021). Oferta (tipos, definición, características y la ley de oferta). Web y Empresas. https://www.webyempresas.com/oferta/

Schencke, et al. (2016), El Rol de la Miel en los Procesos Morfofisiológicos de Reparación de Heridas, 385. Tетисо, Chile. Scielo. https://scielo.conicyt.cl/pdf/ijmorphol/v34n1/art56.pdf

Significados (2017). Consumo Percápita. Consultado el 2 de septiembre dl 2021. https://www.significados.com/per-capita/

Tamaño Diana (2015). Proyecto de factibilidad para la producción de miel de abeja en la parroquia bellavista del canton espindola y su comercilización en loja. (tesis de Pregrado, Universidad Nacional de loja). https://dspace.unl.edu.ec/jspui/bitstream/123456789/10793/1/TESIS\%20DIANA \%20TAMAYO.pdf

Tomas David (2020). Estrategias de Marketing. Ciberklic. https://www.cyberclick.es/numerical-blog/estrategia-de-marketing-que-es-tiposy-ejemplos

Vaca Urbina, G. (2010). Evaluación de proyectos. 6ta. Edición. Mc Granw Hill.

Vásconez Robalino, J (2017). Análisis de los Costos de Producción de la Miel de Abeja en Ecuador. (Tesis pregrado, Universidad San Francisco de Quito). https://repositorio.usfq.edu.ec/bitstream/23000/6604/1/131194.pdf

Vasquez L. (2021). Demanada insatisfecha SCRIBD: https://es.scribd.com/doc/63155301/Demanda-Insatisfecha

Armijos S (19 de febrero 2021). la producción de miel en ecuador atrae mas participantes. Vistazo. https://www.vistazo.com/enfoque/la-produccion-de-mielde-abeja-en-ecuador-atrae-mas-participantes-NGVI223806

\section{LCiencia}




\section{PARA CITAR EL ARTÍCULO INDEXADO.}

Carrillo Parra, E. R., Rojas Oviedo, L. A., \& Novoa Abdo. , T. E. (2021). Determinación de la demanda insatisfecha de miel de abeja en el cantón morona, provincia de Morona Santiago. ConcienciaDigital, 4(3.1), 381-404. https://doi.org/10.33262/concienciadigital.v4i3.2.1845

\section{LCiencia}

El artículo que se publica es de exclusiva responsabilidad de los autores y no necesariamente reflejan el pensamiento de la Revista Conciencia Digital.

El artículo queda en propiedad de la revista y, por tanto, su publicación parcial y/o total en otro medio tiene que ser autorizado por el director de la Revista Conciencia Digital.

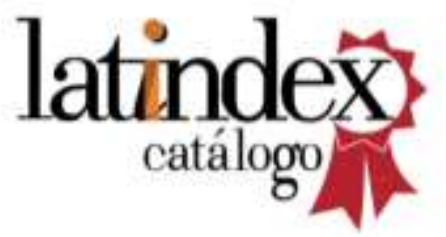

